\title{
RECOVERING OF LOWER ORDER COEFFICIENTS IN FORWARD- BACKWARD PARABOLIC EQUATIONS
}

\author{
S.G. Pyatkov, E.S. Kvich \\ Yugra State University, Hanty-Mansiisk, Russian Federation \\ E-mail: pyatkov@math.nsc.ru
}

We study the issue of recovering a lower order coefficient depending on spatial variables in a forward-backward parabolic equation of the second order. The overdetermination condition is an analog of the final overdetermination condition. A solution at the initial and final moments of time is given. Equations of this type often appear in mathematical physics, for example, in fluid dynamics, in transport theory, geometry, population dynamics, and some other fields. Conditions on the data are reduced to smoothness assumptions and some inequalities for the norms of the data. So it is possible to say that the obtained results are local in a certain way. Under some condition on the data, we prove that the problem is solvable. Uniqueness of the theorem is also described. The arguments rely on the generalized maximum principle and the solvability of theorems of the periodic direct problem. The results generalize the previous knowledge about the multidimensional case. The used function spaces are the Sobolev spaces.

Keywords: inverse problem; final overdetermination; forward-backward parabolic equation; solvability; periodic condition.

\section{Introduction}

Let $G$ be a bounded. The inverse problems is studied in the cylinder $Q=G \times(0, T)$, $S=\Gamma \times(0, T), \Gamma=\partial G$. The problem is stated as follows: find a pair of functions $u(x, t)$ and $\lambda(x)$ satisfying the equation

and the boundary conditions

$$
g(x, t) u_{t}-L u=\lambda(x) u+f(x, t),(x, t) \in Q,
$$

$$
\begin{gathered}
\left.B u\right|_{S}=\varphi(x, t), \\
u(x, 0)=u(x, T)=u_{0}(x) .
\end{gathered}
$$

Here the operator $L$ is of the form $L u=\sum_{i, j=1}^{n} \partial_{x_{j}} a_{i j}(x) u_{x_{i}}-\sum_{i=1}^{n} a_{i}(x) u_{x_{i}}-a_{0}(x) u$ and $B u=u$ or $B u=\sum_{i, j=1}^{n} a_{i j} u_{x_{i}} v_{j}+\sigma(x) u$, where $v_{j}$ are the components of the outer unit normal to $\Gamma$. We assume that the coefficients of the operator $L$ and the boundary operator $B$ as well as the corresponding function spaces are real. The definitions of the function spaces involved can be found, for instance, in [1]. The operator $L$ is elliptic, i. e., there exists a constant $\delta_{0}>0$ such that

$$
\sum_{i, j=1}^{n} a_{i j}(x) \xi_{i} \xi_{j} \geq \delta_{0}|\xi|^{2} \forall \xi \in \mathbb{R}^{n}, x \in G, a_{i j}=a_{j i} \text { for all } i, j .
$$

The inverse problems of the form (1)-(3) in the case of positive function $g(x, t)$ are studied in many articles (see [2-5] and the bibliography therein). In our case the function $g(x, t)$ can change a sign, i. e., we deal with the forward-backward parabolic equation. Equations of this type often appear in mathematical physics, for example, in fluid dynamics while studying fluid motion with alternating coefficient of viscosity, in transport theory while describing the process of particles motion in some environment. Such equations also occur in geometry, population dynamics, and some other fields. Sufficient number of examples is given in [6]. The boundary value problems for equations of the form (1) are studied in many articles (see, for instance, [7, 8]). The inverse problem of finding the right-hand side in (1) is studied in $[9,10,12,13]$. We generalize here the results of the article [13]. Our conditions on the coefficients are more general (in particular, the function in front of the derivative in time can depend on $t)$ and moreover, we prove solvability for an arbitrary $n(n \leq 3$ in [13]). 


\section{Математика}

\section{Preliminaries}

Let $E=L_{2}(G)$. The inner product in $E$ is defined by the equality $(u, v)=\int_{G} u(x) v(x) d x$. Let $D(L)=\left\{v \in W_{2}^{2}(G):\left.B v\right|_{\Gamma}=0\right\}$. The space $H_{1}$ agrees with with $\stackrel{\circ}{2}_{2}^{1}(G)$ in the case of Dirichlet boundary conditions and with $W_{2}^{1}(G)$ in the case of conditions of the third boundary value problem. The space $H_{1}^{\prime}$ is the completion of $E$ in the norm

$$
\|v\|_{H_{1}{ }^{\prime}}=\sup _{w \in H_{1}, w \neq 0}|(v, w)| /\|w\|_{H_{1}},
$$

i. e., it is a negative space constructed on the pair of $H_{1}, E$. The operator $L$ extends to an operator of the class $L\left(H_{1}, H_{1}{ }^{\prime}\right)$ which is the space of linear continuous operator defined on $H_{1}$ with values in $H_{1}{ }^{\prime}$. Define the space

$$
W=\left\{u \in L_{2}\left(0, T ; W_{2}^{1}(G)\right): u_{t}, u_{t t} \in L_{2}\left(0, T ; W_{2}^{1}(G)\right), \partial_{t}^{k} u(x, 0)=\partial_{t}^{k} u(x, T)(k=0,1)\right\} .
$$

where $\partial_{t}^{k}$ are generalized derivatives in the Sobolev sense. By $W_{0}$ we mean the subspace of $W$ of functions satisfying the homogeneous Dirichlet conditions in $S$. Define the norm

$$
\|u\|_{W}=\sum_{i=0}^{2}\left\|\partial_{t}^{i} u\right\|_{L_{2}\left(0, T ; H_{1}\right)} .
$$

Next, we present the condition on the data of the problem. We assume that

$$
\begin{gathered}
a_{i j} \in W_{\infty}^{1}(G), a_{i} \in W_{p}^{1}(G)(i, j=1, \ldots, n) \text { and } a_{0} \in L_{p}(G) ; \\
g, g_{t}, g_{t t} \in L_{\infty}\left(0, T ; L_{p}(G)\right), \partial_{t}^{k} g(x, 0)=\partial_{t}^{k} g(x, T)(k=0,1),
\end{gathered}
$$

where $p>n / 2$ for $n>2$ and $p>1$ for $n \leq 2$;

(i) $f, f_{t}, f_{t t} \in L_{2}\left(0, T ; H_{1}^{\prime}\right), \partial_{t}^{k} f(x, 0)=\partial_{t}^{k} f(x, T)(k=0,1)$;

(ii) $\varphi, \varphi_{t}, \varphi_{t t} \in L_{2}(S), \partial_{t}^{i} \varphi(x, 0)=\partial_{t}^{i} \varphi(x, T) \quad(i=0,1)$ in the case of the Robin boundary conditions and there exists a function $\Phi(x, t) \in W$ such that $\left.\Phi\right|_{S}=\varphi$ in the case of the Dirichlet boundary conditions (this function $\Phi$ exists if, for instance, if $\varphi, \varphi_{t}, \varphi_{t t}, \varphi_{t t t} \in L_{2}\left(0, T ; W_{2}^{1 / 2}(\Gamma)\right.$ ) and $\left.\partial_{t}^{i} \varphi(x, 0)=\partial_{t}^{i} \varphi(x, T) \quad(i=0,1,2)\right)$.

(iii) there exists a constant $\delta_{1}>0$ such that $a_{0}(x)+\alpha g_{t}(x, t)-\frac{1}{2} \sum_{i=1}^{n} a_{i x_{i}}(x)>\delta_{1}>0$ for all $\alpha \in[-1 / 2,3 / 2]$ and a.a. $(x, t) \in Q$;

(iv) $\sigma(x) \in L_{\infty}(\Gamma)$ and $\sigma(x)+\frac{1}{2} \sum_{i=1}^{n} a_{i} v_{i} \geq 0$ for a. a. $x \in \Gamma$ in the case of the Robin boundary conditions.

A pair of functions $u(x, t), \lambda(x)$ is called a solution to problem (1)-(3) if $\lambda(x) \in L_{n / 2}(G)$ for $n>2$, $\lambda(x) \in L_{p}(G)$ with some $p>1$ for $n \leq 2, u \in W$ in the case of Robin boundary conditions, $u-\Phi \in W_{0}$ in the case of the Dirichlet boundary conditions, the conditions (2), (3) holds, and

$$
\int_{G} g u_{t} v+\sum_{i, j=1}^{n} a_{i j} u_{x_{i}} v_{x_{j}}+\sum_{i=1}^{n} a_{i} u_{x_{i}} v+a_{0} u v d Q+\int_{\Gamma} \sigma u v-\varphi v d \Gamma=\int_{G} \lambda u v+f v d G
$$

$\forall v \in H_{1}$, where the integral over $\Gamma$ is absent in the case of the Dirichlet boundary conditions.

Consider an auxiliary problem

$$
\begin{gathered}
M u=g(x, t) u_{t}-L u=f(x, t),(x, t) \in Q, \\
u(x, 0)=u(x, T),\left.B u\right|_{S}=\varphi(x, t) .
\end{gathered}
$$

We can state that 
Theorem 1. Under the conditions (4)-(5), (i)-(iv) there exists a unique solution to the problem (7), (8) such that $u \in W$ in the case of the Robin boundary conditions and $u-\Phi \in W_{0}$ in the case of the Dirichlet boundary conditions. A solution satisfies the estimate

$$
\|u-\Phi\|_{W} \leq c_{0} \sum_{i=0}^{2}\left\|\partial_{t}^{i}(f-M \Phi)\right\|_{L_{2}\left(0, T ; H_{1}\right)}
$$

in the case of the Dirichlet boundary condition and the estimate

$$
\|u\|_{W} \leq c_{0} \sum_{i=0}^{2}\left(\left\|\partial_{t}^{i} f\right\|_{L_{2}\left(0, T ; H_{1}\right)}+\left\|\partial_{t}^{i} \varphi\right\|_{L_{2}(S)}\right)
$$

in the case of the Robin boundary conditions, where the constant $c_{0}$ is some absolute constant $c$ multiplied by the quantity $1 / \min \left(\delta_{1}, \delta_{0}\right)$.

Proof. We can refer to Theorem 3 in [8], where the corresponding result is stated in an abstract form. We need only to check the conditions of this theorem. In the case of the Dirichlet boundary condition Theorem 1 is reduced to Theorem 3 in [8] after the change of variables $u=v+\Phi$. The corresponding check relies on the embedding theorems and the condition of the theorem.

\section{Main results}

In this section we consider the inverse problem in question. To justify the corresponding results below, we employ the generalized maximum principle. So we need to impose some additional conditions on the data.

(v) $g(x, t) \in L_{\infty}(Q), f, f_{t} \in L_{\infty}(Q) ; \varphi, \varphi_{t} \in L_{\infty}(S), u_{0}(x) \in W_{\infty}^{2}(G)$ and there exists a constant $\delta_{2}>0$ such that $u_{0}(x) \geq \delta_{2}$;

(vi) there exists a constant $\delta_{3}>0$ such that $a_{0}(x)+g_{t}(x, t) \geq \delta_{3}>0$ for a.a. $(x, t) \in Q$;

(vii) in the case of the Robin boundary conditions, we have that $\sigma(x) \in C^{1}(\Gamma)$, either $\sigma(x) \geq \delta_{4}>0$ for some constant $\delta_{4}$ and all $x \in \Gamma$ or $\sigma(x) \geq 0$ and $\varphi(x, t) \equiv 0, \varphi(x, t) \in W_{2}^{1 / 4,1 / 2}(S)$, and

$$
\|g(x, 0)\|_{L_{\infty}(G)} R_{1} \leq \operatorname{vraimin}_{G}\left(L u_{0}+f(x, 0)\right), R_{1}=\max \left(\left\|\varphi_{t}\right\|_{L_{\infty}(S)} / \delta_{4},\left\|f_{t}\right\|_{L_{\infty}(Q)} / \delta_{3}\right) ;
$$

(viii) in the case of the Dirichlet boundary conditions we have that $\varphi(x, t) \in W_{2}^{3 / 4,3 / 2}(S)$ and

$$
\|g(x, 0)\|_{L_{\infty}(G)} R_{2} \leq \operatorname{vraimin}_{G}\left(L u_{0}+f(x, 0)\right), R_{2}=\max \left(\left\|\varphi_{t}\right\|_{L_{\infty}(S)},\left\|f_{t}\right\|_{L_{\infty}(Q)} / \delta_{3}\right) .
$$

Theorem 2. Under the conditions (4)-(6), (i)-(viii), there exists a solution $u \in W \cap L_{2}\left(0, T ; W_{2}^{2}(G)\right), \lambda \in L_{\infty}(G)$ to the problem (1)-(3).

Proof. Consider the problem

$$
\begin{gathered}
M_{0} u=g(x, t) u_{t}-L u-\lambda(x) u=f(x, t),(x, t) \in Q, \\
u(x, 0)=u(x, T),\left.B u\right|_{S}=\varphi(x, t),
\end{gathered}
$$

where we assume that $\lambda(x) \leq 0$ a. a. in $G$. In view of Theorem 1 , for a fixed $\lambda \in B_{R}=\left\{\lambda(x) \in L_{p}(G), \lambda(x) \leq 0\right.$ a.e., $\left.\|\lambda\|_{L_{p}(G)} \leq R\right\}$, where $p \geq n / 2$ for $n>2$ and $p>1$ for $n \leq 2$, there exists a unique solution to the problem (11), (12) from the class $W$. This solution satisfies the estimate

$$
\|u-\Phi\|_{W} \leq c_{0} \sum_{i=0}^{2}\left\|\partial_{t}^{i}(f-M \Phi)\right\|_{L_{2}\left(0, T ; H_{1}\right)}+c_{0} \sum_{i=0}^{2}\left\|\lambda(x) \partial_{t}^{i} \Phi\right\|_{L_{2}\left(0, T ; H_{1}\right)}
$$

in the case of the Dirichlet boundary condition and the estimate

$$
\|u\|_{W} \leq c_{0} \sum_{i=0}^{2}\left(\left\|\partial_{t}^{i} f\right\|_{L_{2}\left(0, T ; H_{1}\right)}+\left\|\partial_{t}^{i} \varphi\right\|_{L_{2}(S)}\right)
$$

in the case of the Robin boundary conditions. In view of the embedding $W_{2}^{1}(G) \subset L_{2 n /(n-2)}(G)$ (see [1]) we have (let, $n>2$, for example)

$$
\left|\left(\lambda(x) \partial_{t}^{i} \Phi, v\right)\right| \leq c\|\lambda\|_{L_{n / 2}(G)}\left\|\partial_{t}^{i} \Phi\right\|_{W_{2}^{1}(G)}\|v\|_{W_{2}^{1}(G)}
$$




\section{Математика}

and thus

$$
\left\|\lambda(x) \partial_{t}^{i} \Phi\right\|_{L_{2}\left(0, T ; H_{1}^{\prime}\right)} \leq c\|\lambda\|_{L_{p}(G)}\left\|\partial_{t}^{i} \Phi\right\|_{L_{2}\left(0, T ; W_{2}^{1}(G)\right)} .
$$

Using the conditions on the data and (15), we can rewrite (13) in the form

$$
\|u\|_{W} \leq c_{2} \sum_{i=0}^{2}\left(\left\|\partial_{t}^{i} f\right\|_{L_{2}\left(0, T ; H_{1}\right)}+\|\Phi\|_{W}\right)+c_{3}\|\lambda\|_{L_{p}(G)}
$$

where the constants $c_{2}, c_{3}$ are independent of $\lambda, u$. Differentiate the equality (6) with respect $t$ to and take $v=\left(u_{t}-k\right)^{+}$, with $\left(u_{t}-k\right)^{+}=u_{t}-k$ if $u_{t} \geq k$ and $\left(u_{t}-k\right)^{+}=0$ otherwise. Take $k>0$ and as before assume that $\lambda \in B_{R}$. First, consider the case of the Robin boundary conditions and assume that $\varphi \neq 0$. Integrating with respect $t$ to and by parts we infer

$$
\begin{gathered}
\int_{Q i, j=1}^{n} a_{i j} v_{x_{i}} v_{x_{j}}+\left(a_{0}+\frac{1}{2} g_{t}-\frac{1}{2} \sum_{i=1}^{n} a_{i x_{i}}\right) v^{2}+\left(a_{0}+g_{t}\right) k v d Q+ \\
\int_{S}\left(\sigma+\frac{1}{2} \sum_{i=1}^{n} a_{i} v_{i}\right) v^{2}+\sigma k v-\varphi_{t} v d S=\int_{Q} \lambda u_{t} v+f_{t} v d Q .
\end{gathered}
$$

Here we employ the transformations of the type $a u_{t} v=a\left(u_{t}-k\right) v+a k v=a v^{2}+a k v$. This equality can be rewritten in the form (see the conditions (iv), (vii) and the ellipticity condition)

$$
\int_{Q} \delta_{0}|\nabla v|^{2}+\delta_{1} v^{2}+\delta_{3} k v d Q+\int_{S} \delta_{4} k v-\varphi_{t} v d S \leq \int_{Q} f_{t} v d G
$$

Choosing $k \geq\left\|f_{t}\right\|_{L_{\infty}(Q)} / \delta_{3}$ here and $k \geq\left\|\varphi_{t}\right\|_{L_{\infty}(S)} / \delta_{4}$, we arrive at the inequality

$$
\int_{G} \delta_{0}|\nabla v|^{2}+\delta_{1} v^{2} d Q \leq 0
$$

and, therefore, $v=0$ a. e. or $u_{t}(x, t) \leq k=\max \left(\left\|f_{t}\right\|_{L_{\infty}(Q)} / \delta_{3},\left\|\varphi_{t}\right\|_{L_{\infty}(S)} / \delta_{4}\right)=R_{1}$. Similar arguments applied to a function $-u_{t}$ yield the estimate

$$
\left\|u_{t}(x, t)\right\|_{L_{\infty}(G)} \leq \max \left(\left\|f_{t}\right\|_{L_{\infty}(Q)} / \delta_{3},\left\|\varphi_{t}\right\|_{L_{\infty}(S)} / \delta_{4}\right)=R_{1} .
$$

In the case of the Dirichlet boundary condition an analog of the equality (17) is written as

$$
\int_{Q, j=1}^{n} \sum_{i j} v_{x_{i}} v_{x_{j}}+\left(a_{0}+\frac{1}{2} g_{t}-\frac{1}{2} \sum_{i=1}^{n} a_{i x_{i}}\right) v^{2}+\left(a_{0}+g_{t}\right) k v d Q=\int_{Q} \lambda u_{t} v+f_{t} v d Q
$$

if we take $k \geq\left\|\varphi_{t}\right\|_{L_{\infty}(S)}$. In this case we obtain the estimate

$$
\left\|u_{t}(x, t)\right\|_{L_{\infty}(G)} \leq \max \left(\left\|f_{t}\right\|_{L_{\infty}(Q)} / \delta_{4},\left\|\varphi_{t}\right\|_{L_{\infty}(S)}\right)=R_{2} .
$$

Consider the mapping $A(\lambda)=\left(g(x, 0) u(x, 0)-L u_{0}-f(x, 0)\right) / u_{0}(x)$, where $u$ is a solution to the problem (11), (12). Let $\lambda \in B_{R}$, with $R=\mu^{1 / p}(G)\left(\|g(x, 0)\|_{L_{\infty}(G)} R_{i}+\left\|L u_{0}\right\|_{L_{\infty}(G)}+\|f(x, 0)\|_{L_{\infty}(G)}\right)$, where $i=1$ in the case of the Robin boundary conditions and $i=2$ otherwise, and $\mu(G)$ is the Lebesgue measure of $G$. Demonstrate that this operator $A$ takes a set $B_{R}$ into itself and is compact. Let $\lambda \in B_{R}$. As we have proven, the inequalities (18), (20) hold and the conditions (vii), (viii) imply that $\|A(\lambda)\|_{L_{p}(G)} \leq 0$ a.e. Next, the definition of the quantity and the estimates (14), (20) imply that $\|A(\lambda)\|_{L_{p}(G)} \leq R$, i. e., takes the set $B_{R}$ into itself. The continuity of the mapping $A(\lambda)$ is obvious. Demonstrate that it is compact. Consider a sequence $\lambda_{n}$ with $\lambda_{n} \in B_{R}$. The corresponding sequence of solutions satisfies the estimates (13), (14), (18), (20) and thus the sequence is bounded as well as the sequence $\left\|u_{n t}\right\|_{L_{\infty}(Q)}$. Moreover,

$$
\|A(\lambda)\|_{L_{\infty}(G)} \leq R / \mu^{1 / p}(G)
$$


Fix $p_{0}<2 n /(n-2)$ in the case of $n>2$ and is $p_{0}$ arbitrary if $n \leq 2$. The sequence $\left\|u_{n t}\right\|_{W_{2}^{1}\left(0, T ; W_{2}^{1}(G)\right)}$ is bounded and thus so is the sequence $\left\|u_{n t}\right\|_{C\left([0, T] ; W_{2}^{1}(G)\right)}$. In this case there exists a subsequence $u_{n_{k}}$ such that $u_{n_{k}}(x, 0) \rightarrow v(x)$ in $L_{p_{0}}(G)$ (the embedding theorems). Extracting one more subsequence if necessary we can assume that $u_{n_{k}}(x, 0) \rightarrow v(x)$ a. e. in Lemma 3.2 of Ch.2 in [14] implies that $u_{n_{k}}(x, 0) \rightarrow v(x)$ in any $L_{q}(G)$. We have proven that the mapping is compact. By Schauder fixed point theorem, the equation is solvable on the set $B_{R}$. Consider the equation (11). Since $u \in W$, every summand in this equation belongs $C\left([0, T] ; H_{1}^{\prime}\right)$ to after a possible change on a set of zero measure. So we can take the trace at $t=0$. We obtain that

$$
g(x, 0) u_{t}(x, 0)-L u(x, 0)=\lambda(x) u(x, 0)+f(x, 0) .
$$

The equation $A(\lambda)=\lambda$ can be rewritten as

$$
g(x, 0) u_{t}(x, 0)-L u_{0}(x)=\lambda(x) u(x, 0)+f(x, 0) .
$$

Subtracting these equalities and using the uniqueness theorem for solutions to the problem $L v+\lambda v=0,\left.B v\right|_{\Gamma}=0$, we conclude that $u(x, 0)=u_{0}(x)$. Next, we note that the conditions $u_{t}(x, t) \in L_{\infty}(Q)$ and $u_{t}(x, t) \in C\left([0, T] ; L_{2}(G)\right)$ (we can state even that $u_{t}(x, t) \in C\left([0, T] ; W_{2}^{1}(G)\right)$ ) imply that $u_{t}(x, t) \in L_{\infty}(G)$ for every $t$. Hence, in view of the equality $A(\lambda)=\lambda$ we have that $\lambda \in L_{\infty}(G)$. Rewrite the equation (11) in the form

$$
L u=g u_{t}+\lambda(x) u-f(x, t) \in L_{\infty}(Q) .
$$

In view of the conditions (vii), (viii) and the classical results on solvability of elliptic problem (see [14]), we can conclude that $u \in L_{2}\left(0, T ; W_{2}^{2}(G)\right)$.

In the next theorem we expose the uniqueness conditions. The proof coincides with that in $[13$, Theorem 6]. So we omit it.

Theorem 3. Let the conditions of Theorem 2 hold and

$$
\|g\|_{L_{\infty}(Q)} R_{i} / \delta_{2}<1
$$

where $i=1$ in the case of the Robin boundary conditions and $i=2$ otherwise. Then a solution $(u, \lambda)$ to the problem (1)-(3) from the class pointed out in the claim of Theorem 2 is unique.

Acknowledgement. The authors were supported by the grant on development of scientific schools with participation of young scientists of the Yugra State University.

\section{References}

1. Triebel H. Interpolation Theory, Function Spaces, Differential Operators. North-Holland Mathematical Library, North-Holland Publishing, Amsterdam, 1978, Vol. 18. DOI: 10.1016/s09246509(09)x7004-2

2. Ivanchov M. Inverse problems for equations of parabolic type. Mathematical Studies Monograph Series 10, Lviv, WNTL Publishers, 2003, 238 p. 1999.

3. Kozhanov A.I. Composite Type Equations and Inverse Problems. Berlin, Boston: De Gruyter,

4. Isakov V. Inverse Problems for Partial Differential Equations. Appl. Math. Sci., Berlin, Springer, Cham, 2006, Vol. 127, 344 p. DOI: 10.1007/978-3-319-51658-5

5. Prilepko A.I., Orlovsky D.G., Vasin I.A. Methods for solving inverse problems in Mathematical Physics. New-York: Marcel Dekker, Inc. 1999.

6. Greenberg W., Van der Mee C.V.M., Zweifel P.F. Generalized kinetic equations. Integral Equations and Operator Theory, 1984, Vol. 7, Issue 1, pp. 60-95. DOI: 10.1007/BF01204914.

7. Pyatkov S.G., Popov S.V., Antipin V.I. On solvability of boundary value problems for kinetic operator-differential equations. Integral Equations and Operator Theory, 2014, Vol. 80, Issue 4, pp. 557-580. DOI: 10.1007/s00020-014-2172-7

8. Abasheeva N.L. Razreshimost' periodicheskoy kraevoy zadachi dlya operatornodifferentsial'nogo uravneniya smeshannogo tipa (The solvability of a periodic boundary-value problem 


\section{Математика}

for an operator-differential equation of mixed type). Vestnik NGU. Seriya: Matematika, mekhanika, informatika, 2001, Vol. 1, no. 2, pp. 3-18. (in Russ.).

9. Abasheeva N.L. Determination of a right-hand side term in an operator-differential equation of mixed type. Journal of Inverse and Ill-posed Problems, 2002, Vol. 10, Issue 6, pp. 547-560. DOI: 10.1515/jiip.2002.10.6.547

10. Pyatkov S.G. Razreshimost' lineynoy obratnoy zadachi dlya odnogo klassa singulyarnykh parabolicheskikh uravneniy (The solvability of a linear inverse problem for several classes of singular parabolic equations). Obratnye zadachi i informatsionnye tekhnologii, 2002, Vol. 1, no. 2, pp. 115-123. (in Russ.).

11. Pyatkov S.G. Operator Theory. Nonclassical problems, Utrecht-Boston-Köln-Tokyo: VSP, 2002,348 p.

12. Kaliev I.A., Mugafarov M.F., Fattahova O.V. Inverse problem for forward-backward parabolic equation with generalized conjugation conditions. Ufa mathematical journal, 2011, Vol. 3, no. 2, pp. 33-41.

13. Abasheeva N.L. Some inverse problems for parabolic equations with changing time direction. Journal of Inverse and Ill-posed Problems, 2004, Vol. 12, no. 4, pp. 337-348. DOI: $10.1515 / 1569394042248265$

14. Ladyzhenskaya O.A., Solonnikov V.A., Ural'tseva N.N. Linear and Quasilinear Elliptic Equations. Vol. 46, New-York: Academic Press, 1968, 495 p.

Received April 24, 2018

Bulletin of the South Ural State University Series "Mathematics. Mechanics. Physics" 2018, vol. 10, no. 4, pp. 23-29

УДК 517.956

DOI: $10.14529 / \mathrm{mmph} 180403$

\section{ВОССТАНОВЛЕНИЕ МЛАДШИХ КОЭФФИЦИЕНТОВ В ПАРАБОЛИЧЕСКОМ УРАВНЕНИИ С МЕНЯЮЩИМСЯ НАПРАВЛЕНИЕМ ВРЕМЕНИ}

\section{С.Г. Пятков, Е.С. Квич}

Югорский государственный университет, г. Ханты-Мансийск, Российская Федерация E-mail: pyatkov@math.nsc.ru

Рассматривается обратная задача восстановления младшего коэффициента, зависящего от пространственных переменных, в параболическом уравнении второго порядка с меняющимся направлением времени. Условие переопределения - аналог условия финального переопределения. Решение задается в начальный и конечный момент времени. Уравнения такого типа возникают в математической физике, в задачах гидродинамики, в теории переноса, геометрии, популяционной динамике, и некоторых других областях. Условия на данные сводятся к условиям гладкости и некоторым неравенствам для норм данных. В силу этого можно сказать, что полученные результаты являются в некоторой степени локальными. При выполнении условий на данные доказано, что задача разрешима. Получена также и теорема единственности при несколько более жестких условиях. Задача сводится к операторному уравнению, разрешимость которого устанавливается при помощи априорных оценок и теоремы Лерэ-Шаудера. Доказательства априорных оценок основаны на обобщенном принципе максимума и теоремах о разрешимости периодической задачи. Полученное решение является обобщенным решением и уравнение удовлетворяется в смысле интегрального тождества. Результаты обобщают уже известные на многомерный случай. Используемые функциональные пространства есть пространства Соболева.

Ключевые слова: обратная задача; финальное переопределение; параболическое уравнение с меняющимся направлением времени; разрешимость, периодическое условие. 


\section{Литература}

1. Triebel, H. Interpolation Theory, Function Spaces, Differential Operators, North-Holland Mathematical Library, Vol. 18 / H. Triebel. - North-Holland Publishing, Amsterdam, 1978. DOI: 10.1016/s0924-6509(09)x7004-2

2. Ivanchov, M. Inverse problems for equations of parabolic type / M. Ivanchov // Mathematical Studies Monograph Series 10. - Lviv: WNTL Publishers, 2003. - 238 p.

3. Kozhanov, A.I. Composite Type Equations and Inverse Problems / A.I. Kozhanov. - Berlin, Boston: De Gruyter, 1999.

4. Isakov, V. Inverse Problems for Partial Differential Equations / V. Isakov / Appl. Math. Sci. Berlin: Springer, Cham, 2006. - Vol. 127. - 344 p.

5. Prilepko, A.I. Methods for solving inverse problems in Mathematical Physics / A.I. Prilepko, D.G. Orlovsky, I.A. Vasin. - New-York: Marcel Dekker, Inc., 1999.

6. Greenberg, W. Generalized kinetic equations / W. Greenberg, C.V.M. Van der Mee, P.F. Zweifel // Integral Equations and Operator Theory. - 1984. - Vol. 7. - Issue 1. - P. 60-95.

7. Pyatkov, S.G. On solvability of boundary value problems for kinetic operator-differential equations / S.G. Pyatkov, S.V. Popov, V.I. Antipin // Integral Equations and Operator Theory. - 2014. Vol. 80, Issue 4. - P. 557-580.

8. Абашеева, Н.Л. Разрешимость периодической краевой задачи для операторнодифференциального уравнения смешанного типа / Н.Л. Абашеева // Вестник НГУ. Серия: Математика, механика, информатика. - 2001. - Т. 1, № 2. - С. 3-18.

9. Abasheeva, N.L. Determination of a right-hand side term in an operator-differential equation of mixed type / N.L. Abasheeva // Journal of Inverse and Ill-posed Problems. - 2002. - Vol. 10. - Issue 6. - P. 547-560.

10. Пятков, С.Г. Разрешимость линейной обратной задачи для одного класса сингулярных параболических уравнений / С.Г. Пятков // Обратные задачи и информационные технологии. 2002. - T. 1, № 2. - C. 115-123.

11. Pyatkov, S.G. Operator Theory. Nonclassical problems / S.G. Pyatkov. - Utrecht-Boston-KölnTokyo: VSP, 2002. -348 p.

12. Калиев, И.А. Обратная задача для параболического уравнения с переменным направлением времени с обобщенными условиями сопряжения / И.А. Калиев, М.Ф. Мугафаров, О.В. Фаттахова // Уфимский математический журнал. - 2011. - Т. 3, Вып. 2. - С. 34-42.

13. Abasheeva, N.L. Some inverse problems for parabolic equations with changing time direction / N.L. Abasheeva // Journal of Inverse and Ill-posed Problems. - 2004. - Vol. 12, no. 4. - P. 337-348.

14. Ladyzhenskaya, O.A. Linear and Quasilinear Elliptic Equations. Vol. 46 / O.A. Ladyzhenskaya, V.A. Solonnikov, N.N. Ural’tseva. - New-York: Academic Press, 1968. - 495 p.

Поступила в редакцию 24 апреля 2018 z. 